\title{
Rational selection of hidden epitopes for a molecularly imprinted electrochemical sensor in the recognition of heat-denatured dengue NS1 protein
}

\author{
Matheus Siqueira Silva ${ }^{a}$, Ana Patricia Moreira Tavares ${ }^{\text {b,c }}$, Luiz Felipe Leomil Coelho ${ }^{\mathrm{d}}$, \\ Lígia Ely Morganti Ferreira Dias ${ }^{\mathrm{e}}$, Rosa Maria Chura-Chambi ${ }^{\mathrm{e}}$, Flávio Guimarães da Fonseca ${ }^{\mathrm{f}}$, \\ Maria Goreti Ferreira Sales ${ }^{\text {b, c, } " *}$, Eduardo Costa Figueiredo ${ }^{\text {a," }}$ \\ ${ }^{a}$ Laboratory of Toxicant and Drug Analyses, Federal University of Alfenas, 37130-001, Alfenas, MG, Brazil \\ ${ }^{\mathrm{b}}$ BioMark@ISEP, School of Engineering of the Polytechnic School of Porto, 4200-072, Paranhos, Portugal \\ ${ }^{\mathrm{c}}$ BioMark@UC, Department of Chemical Engineering, Faculty of Sciences and Technology of the University of Coimbra, 3030-790, Coimbra, Portugal \\ ${ }^{\mathrm{d}}$ Laboratory of Vaccines, Department of Microbiology and Immunology, Institute of Biomedical Sciences, Federal University of Alfenas, 37130-001, Alfenas, MG, Brazil \\ ${ }^{\mathrm{e}}$ Center of Biotechnology, Institute of Energetic and Nuclear Research, IPEN-CNEN/SP, 05508-000, São Paulo, SP, Brazil \\ ${ }^{\mathrm{f}}$ Institute of Biological Sciences, Federal University of Minas Gerais, 31270-901, Belo Horizonte, MG, Brazil
}

\section{A R T I C L E I N F O}

\section{Keywords:}

DENV

NS1

Molecularly imprinted polymers

Sensor

Epitope

Label-free

\begin{abstract}
A B S T R A C T
Rational selection of predicted peptides to be employed as templates in molecular imprinting was carried out for the heat-denatured non-structural protein 1 (NS1) of dengue virus (DENV). Conservation analysis among 301 sequences of Brazilian isolates of DENV and zika virus (ZIKV) NS1 was carried out by UniProtKB, and peptide selection was based on in silico data of the conservational, structural and immunogenic properties of the sequences. The selected peptide (from dengue 1 NS1) was synthesized and employed as a template in the electropolymerization of polyaminophenol-imprinted films on the surface of carbon screen-printed electrodes. Heat denaturation of the protein was carried out prior to analysis, in order to expose its internal hidden epitopes. After removal of the template, the molecularly imprinted cavities were able to rebind to the whole denatured protein as determined by electrochemical impedance spectroscopy. This label-free sensor was efficient to distinguish the NS1 of DENV from the NS1 of ZIKV. Additionally, the sensor was also selective for dengue NS1, in comparison with human serum immunoglobulin $\mathrm{G}$ and human serum albumin. Additionally, the device was able to detect the DENV NS1 at concentrations from 50 to $200 \mu^{-1}$ (RSD below 5.04\%, $\mathrm{r}=0.9678$ ) in diluted human serum samples. The calculated LOD and LOQ were, respectively, 29.3 and $88.7 \mu \mathrm{g} \mathrm{L}^{-1}$ and each sensor could be used for six sequential cycles with the same performance.
\end{abstract}

\section{Introduction}

The genus Flavivirus contains more than 70 different viruses classified in 53 species. Yellow fever virus, Japanese encephalitis virus, dengue virus (DENV) and zika virus (ZIKV) are the most frequent human infections caused by these vector-borne diseases. The ZIKV outbreaks of 2013 and 2015, as well as the relation of ZIKV to the increase in fetal and newborn microcephaly cases, reinforced the need for early diagnosis (Gasco and Muñoz-Fernández, 2020). Dengue is a vector-borne disease transmitted to a human host through the bite of an Aedes aegypti or
A. albopictus mosquito infected with DENV. Five significantly different serotypes have been distinguished at an amino acid level, but they still share epidemiologic features and cause similar diseases (Pierson and Diamond, 2013). Langerak et al. (2019) discussed the occurrence of an antibody-dependent enhancement between DENV and ZIKV due to the cross-reactivity of the host antibodies. This cross-reactivity occurs due to the high degree of homology observed between these species. The first licensed dengue vaccine, CYD-TDV, still offers risks for seronegative patients and this lack of preventive measures emphasizes the need for a precise diagnosis (Wilder-Smith, 2018).

\footnotetext{
* Corresponding author.

** Corresponding author. BioMark@ISEP. School of Engineering of the Polytechnic School of Porto, 4200-072, Paranhos, Portugal.

E-mail addresses: goreti.sales@eq.uc.pt (M.G. Ferreira Sales), eduardo.figueiredo@unifal-mg.edu.br (E. Costa Figueiredo).
} 
Confirmatory diagnosis of dengue and zika is difficult, especially in countries endemic for other arboviruses. Molecular diagnosis is not always available and reference laboratories may be overloaded during epidemics. Furthermore, DENV and ZIKV cross-react immunologically with other flaviviruses, making the interpretation of serological results complex (Braga et al., 2017). Non-structural protein 1 (NS1; $\approx 46 \mathrm{kDa}$ ) is a soluble glycoprotein produced by flaviviruses and implemented either for early diagnosis in the first days of fever (when specific IgM is not detectable), or at up to 9 days of infection (when RT-PCR for DENV RNA is negative). It is secreted as a hexameric lipoparticle, circulating in the serum of infected patients at concentrations up to $50 \mathrm{mg} \mathrm{L}^{-1}$. This biomarker may be complexed with the host's antibody, especially during secondary infections (Blacksell, 2012; Darwish et al., 2015; Gelanew et al., 2015).

Biosensors with natural antibodies have been used for detecting proteins (e.g. NS1) (Anusha et al., 2019; Khristunova et al., 2020). However, recognition elements of a biological nature (e.g.: antibodies, enzymes and histones) are costly and lack stability, and have been replaced by artificial elements such as molecularly imprinted polymers (MIPs) (Arshad et al., 2020; Tai et al., 2005). MIP materials are able to mimic the affinity of antibodies to antigens, reproducing a template molecule with structural conformation, regarding the position of functional groups and steric hindrance. Several applications of molecular imprinting technology have been extensively reviewed in the literature (Ertürk and Mattiasson, 2017; Frasco et al., 2017; Ramanavicius and Ramanavicius, 2021; Saylan et al., 2017; Silva et al., 2020).

The polymerization of electroactive compounds over the working electrode allows the blending of molecular imprinting technology with electrochemical transduction to produce a label-free, easily handled fast analytical device. According to Erdössy et al. (2016), cyclic voltammetry is one of the most widespread electropolymerization techniques for the preparation of MIPs to recognize proteins. Electropolymerization enables fine tuning of the morphology, homogeneity, conductivity, thickness and overoxidation level of the formed polymer layer with molecular imprints (Ramanavicius and Ramanavicius, 2021). The density and thickness of MIPs can be tuned by varying the number of scan cycles, the scan rate and the concentration of the monomeric mixture elements, restricting the formation of binding sites to the MIP film surface.

Proteins displays complex and flexible structures, which may fold as a response to minimal changes in the physicochemical conditions of a system. While this flexibility is a natural tool to assure a proper turnover and regulate protein functions, it may also be an undesirable factor for subsequent protein recognition, which occurs in an imprinted rigid structure. In this regard, epitope imprinting simplifies the process. It is a well-known approach to recognizing the external epitopes of proteins (Mujahid et al., 2018; Zahedi et al., 2016), formerly applied even to NS1 detection (Tai et al., 2005). The advantage comes from the use of a template of reduced size, causing it to be less susceptible to conformational changes during synthesis, and avoiding the conformational packing that assembles the tertiary structure of the protein. It is highly desirable to manage the template in order to keep its conformation as similar as possible to that in the target analyte. The use of water-soluble monomers helps to avoid the denaturation of protein templates, and a more stable template can be developed by the epitope imprinting approach with a rational design.

The imprinting of an internal epitope was introduced by Bossi et al. (2012) based on the recognition of epitopes produced by specific enzymatic digestion of a protein (analyte). An internal epitope represents a section of the sequence that is less accessible to superficial interactions, which becomes exposed after the protein unfolds. This disruption of the tertiary structure of the protein uncovers the epitopes and aids the recognition element to anchor the imprinted fragment of the analyte. Gelanew, Poole-Smith and Hunsperger (2015) developed monoclonal antibodies against monomeric heat-denatured NS1, characterizing the epitopes by peptide scan. A denaturation step prior to analysis improved both sensitivity, since it released epitopes previously blocked by antigen-antibody immunocomplexes, and selectivity, since the denaturation process disrupted the tertiary structure of NS1, exposing linear epitopes and lowering steric hindrance.

This work employed a rational design to select a predicted epitope (template for MIP) based on in silico data of conservational, structural and immunogenic properties, as well as to build and test a chemosensor capable of differentiating the NS1 proteins from DENV and ZIKV. Heatmediated denaturation of NS1 was carried out prior to analyses. This denaturation unfolds the tertiary structure in a quick way without the use of enzymes or chemical agents. Moreover, exposure of internal fragments of the sequence allowed the selection of hidden epitopes by the rational design, avoiding competition with antibodies for the binding site. It also allows the choice of highly distinct regions among different species, thereby allowing differential diagnosis of infectious diseases.

\section{Experimental}

\subsection{Reagents and solutions}

All chemicals were of analytical grade, and ultrapure water was obtained from a Milli-Q water purification system (resistivity 18.2 M $\Omega$ $\mathrm{cm})$. Potassium hexacyanoferrate II $\left(\mathrm{K}_{4}\left[\mathrm{Fe}(\mathrm{CN})_{6}\right] \cdot 3 \mathrm{H}_{2} \mathrm{O}\right)$ and potassium hexacyanoferrate III $\left(\mathrm{K} 3\left[\mathrm{Fe}(\mathrm{CN})_{6}\right]\right)$ were obtained from Riedel de Haën (Charlotte, NC, USA). Potassium chloride $(\mathrm{KCl})$ was obtained from Merck (Kenilworth, NJ, USA) and phosphate-buffered saline (PBS) tablets were obtained from Amresco (Dallas, TX, USA). 3-Aminophenol (3$\mathrm{AP}$ ) and proteinase $\mathrm{K}$ were from Sigma-Aldrich (San Louis, MO, USA). Commercial carbon screen-printed electrodes (C-SPEs; Ref. C-110) were purchased from DropSens (Comunidad Valenciana, Spain).

The peptide sequence template ${ }^{28}$ WTEQYKFQA ${ }^{36}$ was purchased from GenScript (Piscataway, NJ, USA). Recombinant dengue virus serotype 1 (DENV-1) NS1 protein hexamer was purchased from the Native Antigen Company (Oxford, UK). The NS1 from dengue virus serotype 2 (DENV-2) was produced in a procaryotic vector. Briefly, the protein's coding sequence was obtained from public repositories and codon-adapted for expression in Escherichia coli. The gene was commercially obtained, subcloned in a pET21 expression vector and used to transform BL21(DE3) cells. Expression was induced by adding IPTG to the culture medium, and the expressed protein was visualized in PAGE and purified by affinity chromatography in an AKTA system (GE Healthcare). ZIKV NS1 was expressed in E. coli as inclusion bodies (NS1IB). NS1 was solubilized from the NS1-IB utilizing high pressure and $\mathrm{pH}$ 11.0 in the presence of arginine, as described by Rosa Da Silva et al. (2018). Immunoglobulin G from human serum (IgG) and human serum albumin (HSA) were purchased from Sigma-Aldrich (San Louis, MO, USA).

\subsection{Apparatus}

The protein heat denaturation was carried out in a J. P. Selecta Thermobloc (Barcelona, Spain). Electrochemical data were obtained using an Metrohm Autolab PGSTAT128N potentiostat/galvanostat with a FRA32M module (Barendrecht, Netherlands), controlled by Nova 2.1.3 software. The SPEs were connected to the equipment through a Metrohm DropSens DRP-DSC box connector (Ovedo, Spain). Raman readings were carried out in a modular Raman system composed of an Olympus B-X41 microscope (Tokyo, Japan), a Horiba iHR550 monochromator (Darmstadt, Germany) and a $532 \mathrm{~nm}$ solid state laser (B\&W Tek, Newark, Denmark).

\subsection{Rational selection of the template}

NS1 sequences from Brazilian isolates of DENV (serotypes 1, 2, 3 and 4) and ZIKV were prospected at UniProtKB. These sequences were aligned by the ClustalW algorithm of MEGA software 10 (https://www. 
megasoftware.net/). To select the desirable structural features of the template, the sequences were analyzed by the prediction algorithms Emini Surface Accessibility, Karplus \& Schulz Flexibility and BepiPred Linear Epitope Prediction, available in the Immune Epitope Database and Analysis Resource (IEDB; https://www.iedb.org/). The region was then selected, and immunogenicity was evaluated by other IEDB tools, MHC I Binding and MHC II Binding, based on the Stabilized Matrix Method algorithm.

\subsection{Chemosensor construction and characterization}

Prior to modification, the commercial C-SPEs were washed thoroughly with ultrapure water, followed by electrochemical cleaning from -0.3 to $1.5 \mathrm{~V}$, at a rate of $50 \mathrm{mV} \mathrm{s}^{-1}$ in a $0.5 \mathrm{~mol} \mathrm{~L}^{-1}$ sulfuric acid solution for 10 cycles. The electrodes were washed again with ultrapure water.

Epitope-imprinted films were deposited on C-SPEs by electropolymerization using a mixture of $2.5 \mathrm{mmol} \mathrm{L}^{-1} 3$-AP and $250 \mu \mathrm{mol} \mathrm{L}^{-1}$ ${ }^{28}$ WTEQYKFQA $^{36}$ peptide, prepared in $10 \mathrm{mmol} \mathrm{L}^{-1} \mathrm{PBS}$ at $\mathrm{pH}$ 7.4. Electropolymerization was achieved by collecting 10 cyclic voltammograms between 0 and $0.38 \mathrm{~V}$ at the scan rate of $25 \mathrm{mV} \mathrm{s}^{-1}$. The SPEs were washed thoroughly with water and subjected to 5 more cyclic voltammograms, in $10 \mathrm{mmol} \mathrm{L}^{-1} \mathrm{PBS}$ (pH 7.4) under the same conditions, in order to stabilize the formed film. A similar procedure without addition of the peptide template was implemented to act as control material for non-specific binding, called non-imprinted polymer (NIP). The resulting modified electrodes were thoroughly rinsed with ultrapure water to remove the remains of any reagent, dried under a nitrogen flow and stored properly at room temperature.

Template removal was performed in both MIP and NIP by treatment with $0.5 \mathrm{~g} \mathrm{~mL}^{-1}$ proteinase $\mathrm{K}$ solution prepared in $10 \mathrm{mmol} \mathrm{L}^{-1} \mathrm{PBS}$ ( $\mathrm{pH}$ 7.4) for $1 \mathrm{~h}$ at $25{ }^{\circ} \mathrm{C}$. After this, 25 more cyclic voltammograms were carried out in $10 \mathrm{mmol} \mathrm{L}^{-1} \mathrm{PBS}$ (pH 7.4) using the same conditions as for polymerization, followed by subsequent gentle washing with ultrapure water and storage for further use.

Raman readings were obtained immediately after electrode cleaning, polymerization and template removal. The spectra were collected in the regions of interest between 900 and $1950 \mathrm{~cm}^{-1}$, and 2350 and 3250 $\mathrm{cm}^{-1}$ of Raman shift.

\subsection{Electrochemical assays}

Electrochemical measurements were obtained with equimolar amounts of $5.0 \mathrm{mmol} \mathrm{L}^{-1}\left[\mathrm{Fe}(\mathrm{CN})_{6}\right]^{3-/ 4-}$ as a redox couple, using 10 mmol L ${ }^{-1}$ PBS ( $\mathrm{pH}$ 7.4) as supporting electrolyte. Electrochemical impedance spectroscopy (EIS) assays were performed in an open circuit potential, with a sinusoidal potential perturbation amplitude of $0.01 \mathrm{~V}$ with readings collected over the frequency range $0.01-10,000 \mathrm{~Hz}$. Data were gathered at room temperature. Between each measurement, the electrodes were gently rinsed in ultrapure water and dried under flowing nitrogen gas.

Chemical modifications to each electrode were followed by checking the variations in the charge transfer resistance $\left(R_{c t}\right)$ from EIS. Experimental data were fitted with NOVA 2.1.3 software, and the Randles circuit model (with Warburg diffusion) was adopted as an equivalent circuit for the present system.

The rebinding experiments were evaluated by consecutive EIS readings. After stabilization in PBS, the sensor was submitted to successive incubations with rising concentrations of the analyte, being gently rinsed with water before and after the incubation. The last stabilization reading was set as the blank. The response for the rebinding experiments was normalized by the last stabilization response and defined as relative $R_{c t}\left(R_{c t(x)} / R_{c t(s t a b i l i z e d)}\right)$.

\subsection{NS1 rebinding}

The system response to denatured NS1 was monitored by recording calibration curves. This procedure was initiated by the incubation of increasing concentrations of heat-denatured NS1 (from 25 to $200 \mu \mathrm{g} \mathrm{L}^{-1}$, in $10 \mathrm{mmol} \mathrm{L}^{-1} \mathrm{PBS}, \mathrm{pH}$ 7.4) on the polymer sensing films, for $30 \mathrm{~min}$. This was followed by washing with water to remove any loosely bound materials that could have been adsorbed to the surface.

\section{Results and discussion}

\subsection{Rational selection of the template}

The following characteristics are desirable for a template epitope: (1) a similar isoelectric point to the protein; (2) the avoidance of areas susceptible to post-translational modification; (3) a limited sequence size (generally from 7 to 15 amino acids) to allow some degree of selectivity, but not so small that it may hinder recognition of the target template; (4) a conserved sequence among distinct isolates of the same species which differs from other related organisms, in this case, other related flaviviruses; (5) weak immunogenicity, to evade competition with host antibodies. For the template epitope selection, our starting point was the LD2 epitope $\left({ }^{24}\right.$ VHTWTEOYK $\left.{ }^{32}\right)$, located by Falconar et al. (1994). According to them, LD2 shows partial dependence upon conformation, indicating that flanking sequences and regions may contribute to the recognition of sequence composition and conformation. Gelanew, Poole-Smith and Hunsperger (2015) mapped a monoclonal antibody to the sequence ${ }^{25}$ EVHTWTEQYKFQADSP ${ }^{39}$ and proved its capability to bind to monomeric and even to hexameric forms, suggesting that the forward side (from 33 to 39 ) of the sequence is a less restricted region. To achieve these characteristics for a molecular imprinting template, we aimed to reduce the ${ }^{25}$ EVHTWTEQYKFQADSP $^{39}$ fragment to a nine-residue template. To do so, conservation analysis was performed, prospecting 67 and 20 NS1 sequences for DENV-1 and ZIKV, respectively. The conservation of a sequence is a pivotal element for specificity in epitope imprinting protocols. The sequence chosen should be conserved among different isolates of the target species and serotype. For the conservation analysis, we also prospected 137, 62 and 15 NS1 sequences of DENV-2, -3 and -4, respectively. The reviewed sequences P27909 (DENV-1 - strain Brazil/97-11/1997) and Q32ZE1 (ZIKV - strain Mr 766) were taken as references for the conservation analysis (Table S1) and structural prediction (Figs. S1-S3). Immunogenicity was determined as the probability of the selected sequence being recognized by $\mathrm{T}$ cells. The region ${ }^{25}$ EVHTWTEQYKFQADSP $^{39}$ was analyzed by the MHC I Binding and MHC II Binding tools with the recommended preset of alleles for each tool. The binding affinity thresholds $\left(\mathrm{IC}_{50}\right)$ for each allele of MHC Classes I and II predicted by the Stabilized Matrix Method are depicted in Fig. S4. The predicted $\mathrm{IC}_{50}$ values were all below the cut-off values of $500 \mathrm{nmol} \mathrm{L}^{-1}$, for MHC class I, and $1000 \mathrm{nmol} \mathrm{L}^{-1}$, for class II. So, the region exhibited no alert of immunogenicity.

Since the template is not immobilized, its disposition in the polymerization mixture is random. The Karplus \& Schulz Flexibility Prediction algorithm determines the flexibility of an amino acid residue based on the B factor of $\alpha$-carbons (Karplus and Schulz, 1985). Flexible regions (Fig. S1) should be avoided to obtain more uniform imprinted cavities. Nonetheless, the template peptide should not be rigid, to facilitate its removal after imprinting. Linear regions (Fig. S2) may also contribute to template removal since non-linear epitopes are more susceptible to becoming entangled with the polymeric network after polymerization. The BepiPred Linear Epitope Prediction combinatorial model provides a score for each residue of the sequence based on Hidden Markov models (Larsen et al., 2006). After the heat denaturation, we were aiming for an accessible region (Fig. S3) to perform easier rebinding to the imprinted cavity. A residue is considered accessible when it exhibits an area of more than $20 \AA$ accessible to water. The 
Emini Surface Accessibility Prediction algorithm determines the accessibility of a residue by empirical comparison (Emini et al., 1985). We avoided the flexibility of residues 37, 38 and 39 (Fig. S1) and selected as a template the accessible and partially linear (Figs. S3 and S2, respectively) nine-residue region ${ }^{28}$ WTEQYKFQA $^{36}$ (Fig. 1). The selected region remains conserved in all the isolates of serotypes 1 and 3 tested, differing from all isolates of serotypes 2 and 4 at residue 36 (exchanging an alanine for a proline). At least $66.66 \%$ of this sequence differs from that of the $20 \mathrm{ZIKV}$ isolates tested. The conservation analysis results are available in Table S1.

\subsection{Electrochemical behavior of the mixture}

First, the electrochemical behavior of each component of the polymerization mixture was evaluated in PBS from 0 to $1.5 \mathrm{~V}$, in order to identify the best potential range in which to carry out the electropolymerization, while keeping the template intact. The results obtained are displayed in Fig. 2, showing monomers and epitope tested separately in the same conditions. Overall, the monomer 3-aminophenol exhibits a distinct electroactive behavior beginning at $0.2 \mathrm{~V}$ with a peak around $0.5 \mathrm{~V}$. The ${ }^{28} \mathrm{WTEQYKFQA}{ }^{36}$ peptide remains inactive up to $0.4 \mathrm{~V}$, from which point it starts showing electroactivity. To confirm that this electroactivity was really from the peptide, cyclic voltammetry of the PBS solution alone was carried out in the same potential range. It was possible to observe that the current from the peptide increased and it became higher than that of PBS from potentials greater than $0.4 \mathrm{~V}$, thereby confirming the electroactivity of the peptide.

Based on these observations, the range of $0-0.38 \mathrm{~V}$ was selected for the polymerization. This range would avoid any chemical modifications of the template, by promoting its oxidation under higher potentials. In addition to this, any oxidation of the template could contribute to its participation in the polymerization stage, which in turn would entrap the peptide within the polymeric network by covalent binding, thereby limiting its removal.

\subsection{Assembly of the recognition element}

Each step of the sensor construction is exhibited in Fig. 3. Pretreatment of the C-SPE with $\mathrm{H}_{2} \mathrm{SO}_{4}$ promotes the formation of an oxidized surface. Despite the lower conductivity, this modified surface aids adhesion of the polymer film to the carbon ink of the working electrode. After activation of the surface, the system is rinsed with water and the polymerization is performed, promoting a similar increase in $\mathrm{R}_{\mathrm{ct}}$ in both MIP and NIP films. The formed polyaminophenol network promoted an insulating effect directly proportional to the thickness and

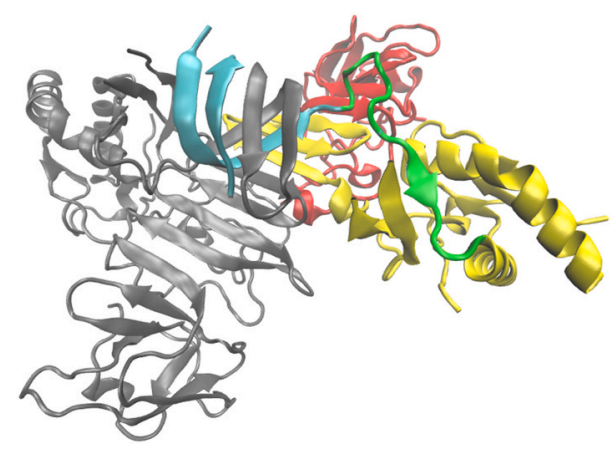

Fig. 1. NS1 dimer structure of DENV-2 (4O6B) with one subunit in gray, the ${ }^{28}$ WTEQYKFQA $^{36}$ fragment highlighted in green and the other colored by domain (cyan, $\beta$-roll; yellow, wing; and red, central $\beta$-ladder). Atomic coordinates and structure factor files obtained in RCSB Protein Data Bank (PDB) under the accession code 4O6B from Akey et al. (2014). (For interpretation of the references to color in this figure legend, the reader is referred to the Web version of this article.)

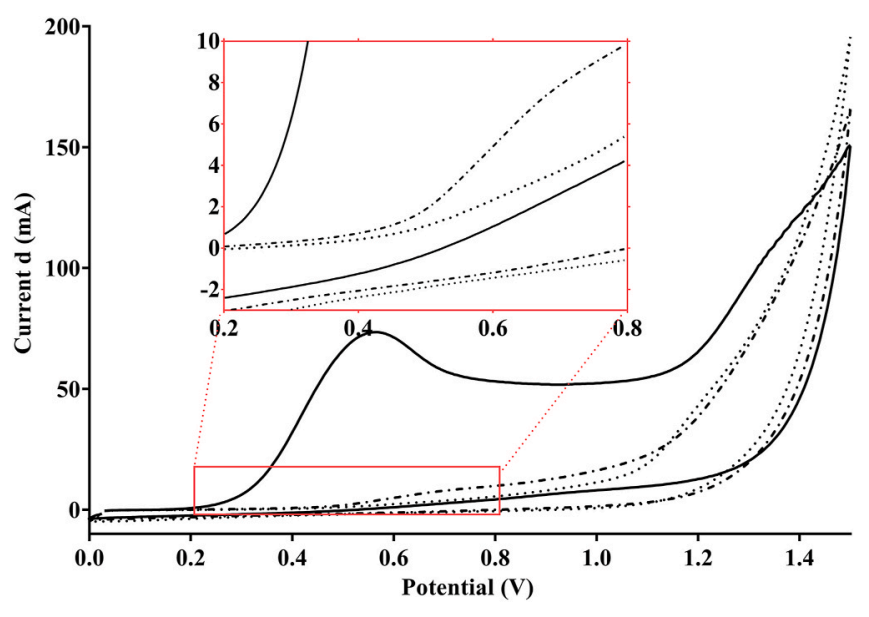

Fig. 2. Electroactivity of each component of the polymer mixture by cyclic voltammetry. First cycle for pure $10 \mathrm{mmol} \mathrm{L}^{-1} \mathrm{PBS}$, pH 7.4 (solid); $2.5 \times 10^{-3}$ mol L ${ }^{-1}$ 3-aminophenol (@/) prepared in $10 \mathrm{mmol} \mathrm{L}^{-1} \mathrm{PBS}, \mathrm{pH} 7.4$; and 250 $\mu \mathrm{mol} \mathrm{L}{ }^{-1}{ }^{28} \mathrm{WTEQYKFQA}^{36}$ peptide (//-) prepared in $10 \mathrm{mmol} \mathrm{L}^{-1} \mathrm{PBS}$, $\mathrm{pH} 7.4$.

density of the film. The lower $\mathrm{R}_{\mathrm{ct}}$ observed in the MIP may be correlated to the presence of the functional groups of the ${ }^{28}$ WTEQYKFQA ${ }^{36}$ fragment, reducing the insulating effect of the system. The thickness of the resulting films was calculated by coulometric analysis after formation of the film (Ribeiro et al., 2017). The following equation was employed:

$d=\frac{M Q}{F A \rho}$

where $d$ represents the thickness of the film, $M$ is the molecular mass of 3-aminophenol monomer $\left(1.195 \mathrm{~g} \mathrm{~cm}^{-3}\right), Q$ is the total charge transferred during the electropolymerization - calculated by integration of the voltammogram by Nova Software, $F$ is the Faraday constant $(9.6485$ $\left.\times 10^{-4} \mathrm{C} \mathrm{mol}^{-1}\right), A$ is the surface area of the working electrode $(0.126$ $\mathrm{cm}^{2}$ ) and $\rho$ is the density of the polymer, considered the same as for 3aminophenol $\left(1.195 \mathrm{~g} \mathrm{~cm}^{-3}\right)$. Charges of 380.40 and $232.43 \mu \mathrm{C}$ and a respective estimated thickness of 24.54 and $17.51 \mathrm{~nm}$ were obtained for NIP and MIP, respectively.

The system was gently rinsed with water, and the removal began by incubation with proteinase $\mathrm{K}$. A rise in $\mathrm{R}_{\mathrm{ct}}$ can be observed for both MIP and NIP after 30 min of enzymatic digestion at room temperature. Since the predominant cleavage sites of the enzyme are peptidic bonds near aromatic rings, it should not affect the NIP so much. But Khan et al. (2016) reported that the enzyme may partially digest p-aminophenol films due to the similarity between these cleavage sites and the polymeric network (plenty of oxygen and nitrogen-related groups). Since the enzyme may promote modification of the polymeric film and occasionally damage the imprinted cavities, the analytical performance of the sensor was evaluated for single use.

To finish the removal, the system was gently rinsed with water and the cleaning stage was completed by performing $25 \mathrm{CV}$ cycles in PBS, to remove any digested fragments that could remain adsorbed. After these cycles, the NIP sensor remained almost unchanged, while the MIP $\mathrm{R}_{\mathrm{ct}}$ dropped out to levels below those at the post-polymerization stage. This can be justified by the removal of the template, leaving behind the imprinted cavities to be filled with the reading probe, reducing the impedance of the surface.

\subsection{Raman spectroscopy characterization}

The Raman spectra (Figs. S5 and S6) illustrate three peaks generally obtained in carbon materials, found at around 1370, 1580 and 2700 $\mathrm{cm}^{-1}$ Raman shift, typically known as G, D and 2D bands, respectively. 
NIP

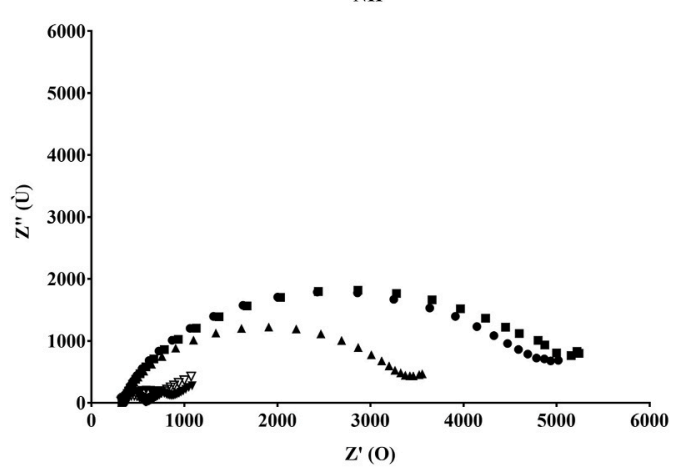

MIP

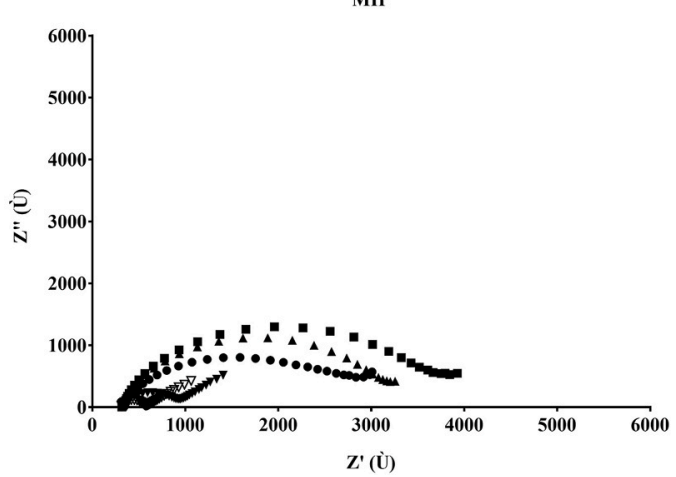

Fig. 3. EIS readings for bare electrode $(\nabla)$, cleaning treatment $(\boldsymbol{\nabla})$, polymerization $(\boldsymbol{\Delta})$, treatment with proteinase K $(\square)$, removal step by cyclic voltammetry $(\boldsymbol{Q})$

The $\mathrm{G}$ band corresponds to the bond stretching of all pairs of $\mathrm{sp}^{2}$ carbons in both rings and chains. The $\mathrm{D}$ peak originates from the breathing modes of the six-atom rings of graphene and requires a defect for its activation. The intensity is proportional to the disorder of the network. The 2D band corresponds to the second-order zone-boundary phonons or an overtone of the D band. The second-order peak around $2950 \mathrm{~cm}^{-1}$ can be assigned to a combination of D and G bands (D $+G$ ) (Bulusheva et al., 2008; Ferrari et al., 2006; Kawashima and Katagiri, 1995; Roscher et al., 2019). The $\mathrm{I}_{\mathrm{D}} / \mathrm{I}_{\mathrm{G}}$ ratio is used to infer defects in carbon materials. The NIP (Fig. S5) displayed $\mathrm{I}_{\mathrm{D}} / \mathrm{I}_{\mathrm{G}}$ ratios of $0.9723>0.9154>0.9064$ for cleaning $>$ polymerization $>$ removal. This means that the surface exhibited fewer defects after each of the monitored steps. The shape of the $2 \mathrm{D}$ band is affected by doping and stress. The $2 \mathrm{D}$ band of the NIP built up during the polymerization, reflecting the presence of the aminophenol N- and O- groups in the aromatic rings. The MIP (Fig. S6) displayed $\mathrm{I}_{\mathrm{D}} / \mathrm{I}_{\mathrm{G}}$ ratios of $0.9723>0.9035>0.9549$ for cleaning $>$ polymerization $>$ removal. Similar behavior of MIP and NIP can be observed after the polymerization, but the increase of the $\mathrm{I}_{\mathrm{D}} / \mathrm{I}_{\mathrm{G}}$ ratio for MIP after the removal step indicates more defects and corroborates the formation of the imprinted cavities. Furthermore, the lesser intensity of the 2D band in the MIP after the removal step indicates a minor contribution from doped content in the network and matches with the removal of the template.

\subsection{Analytical performance of the system}

The analytical performance of the sensor was first evaluated by EIS, testing the binding of the ${ }^{28}$ WTEQYKFQA ${ }^{36}$ fragment to MIP and NIP sensors. The peptide was previously heat-denatured to reproduce the closest conformation of the denatured protein to be further analyzed. The response was calculated as relative $R_{c t}$. As observed in Fig. 4, MIP displays a rise of $R_{c t}$ directly proportional to the increase in peptide concentration, while NIP exhibits random behavior with increasing concentrations of peptide. This could mean that rebinding of the peptide is dominated by the imprinted cavities of the polymer. The imprinting factor was calculated as the ratio of the signals obtained at the epitope rebinding for the MIP and NIP at the higher concentration of the analyte. The averages of relative $\mathrm{R}_{\mathrm{ct}}$ found for the epitope rebinding at $10^{-4} \mathrm{~mol}$ $\mathrm{L}^{-1}$ were $13.99 \%$ and $1.10 \%$ for MIP and NIP, respectively. So, the calculated IF was 12.72 .

Knowing that the cavities were successfully imprinted, the next step consisted of evaluating the sensor's ability to bind to the whole protein and estimate the contribution of the heat denaturation to the affinity of the polymer (Fig. 5a and b). Overall, the folded protein displayed a random behavior (Fig. 5a), with slight variations in $R_{c t}$ throughout the whole concentration range of the calibration. This small variation reflected the weak binding affinity of this folded protein to the SPE surface. In contrast, the heat-denatured NS1 displayed the opposite behavior (Fig. 5 b). There were significant changes in $\mathrm{R}_{\mathrm{ct}}$, which were concentration-dependent. In MIP, the $\mathrm{R}_{\mathrm{ct}}$ decreased proportionally to the denatured protein concentration, attributed to the interaction of exposed residues of the denatured protein with the reading probe. It is known that in faradaic EIS biosensors, the $\mathrm{R}_{\mathrm{ct}}$ may increase (in most cases) or decrease in response to target binding (Luo and Davis, 2013), justifying this reverse behavior between the epitope and the protein. Supporting the huge sensitivity observed, Gelanew et al. (2015) reported that their monoclonal antibodies exhibited less activity against hexameric and dimeric forms of NS1 and amplified performance against heat-denatured monomeric forms, proving that exposure of the epitope boosts the effectiveness of the recognition. The results obtained from the calibration curve plotted by NS1 concentration against $R_{c t}$ showed that the sensor had a good analytical performance with linear correlation $(\mathrm{r}$ $=0.9893)$ and RSD below $5.58 \%(n=3)$ from 10 to $200 \mu \mathrm{g} \mathrm{L}^{-1}$. Additionally, the sensor exhibited an LOD and LOQ of 13.9 and $42.2 \mu \mathrm{g} \mathrm{L}^{-1}$, respectively. The bulky body of the 352 residues of the protein increased the intensity of the response signal and reduced drastically the RSD,
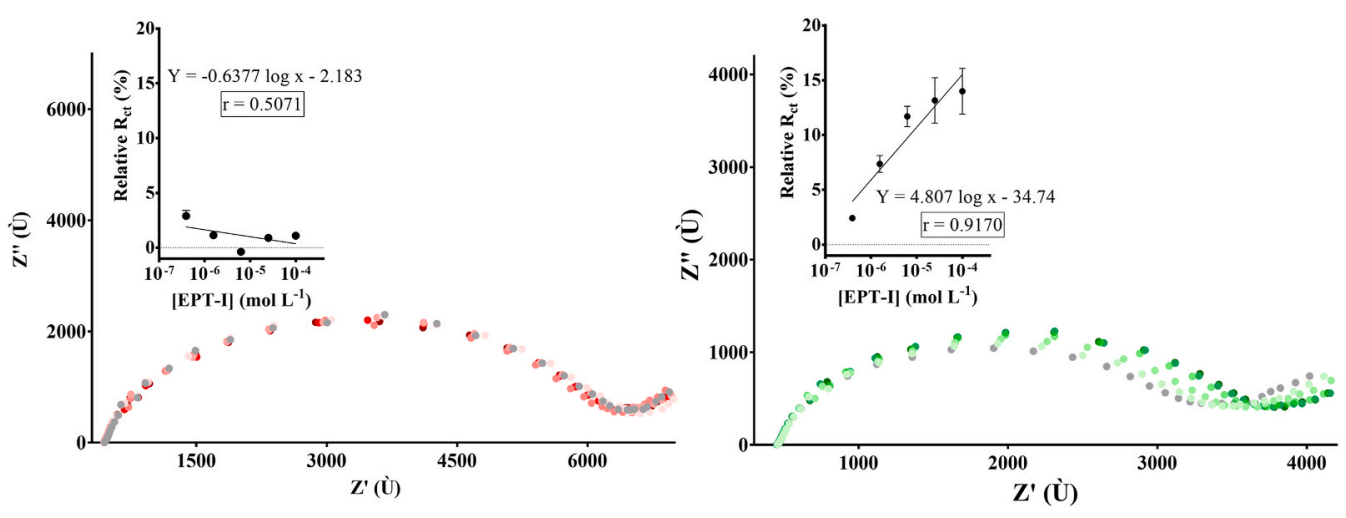

Fig. 4. EIS calibration curves for ${ }^{28}$ WTEQYKFQA ${ }^{36}$ fragment rebinding in NIP (red) and MIP (green). The reading for stabilized sensor (gray) is followed by that for rising concentrations of the peptide, from $3.91 \times 10^{-7}$ to $1 \times 10^{-4}$ mol L $\mathrm{L}^{-1}$, with RSD of up to $17.35 \%$ and $34.41 \%(\mathrm{n}=3)$ for MIP and NIP, respectively. A semilog linear regression was performed between the concentration of epitope $\left(\mathrm{mol} \mathrm{L}^{-1}\right)$ and relative

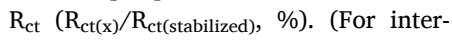
pretation of the references to color in this figure legend, the reader is referred to the Web version of this article.) 

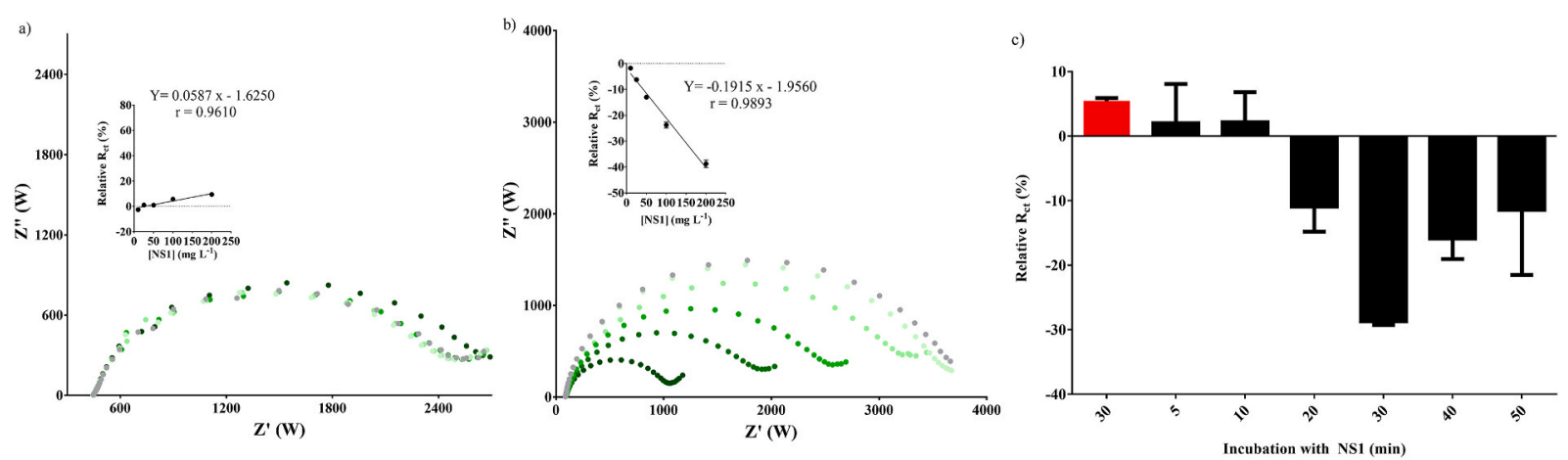

Fig. 5. EIS calibration curves for rebinding with folded (a) and heat-denatured (b) DENV-1 NS1 protein. The reading for stabilized sensor (gray) is followed by that for rising concentrations of the protein, from 25 to $200 \mu \mathrm{g} \mathrm{L}^{-1}$, with RSD below $5.24 \%(\mathrm{n}=3)$. The behavior of the sensor was also evaluated against increasing duration of incubation (c) with $100 \mu \mathrm{g} \mathrm{L}^{-1}$ folded (red) and previously heat-denatured NS1 (black). (For interpretation of the references to color in this figure legend, the reader is referred to the Web version of this article.)

when compared to the nine-residues epitope rebinding.

To investigate the effect of the heat denaturation, the sensor was submitted to incubations of increasing duration with the previously heat-denatured NS1 (Fig. 5c). Due to having cysteine residues that form disulfide bonds, NS1 was able to form highly stable homodimers, around 30 min after the synthesis (Winkler et al., 1988, 1989). The behaviors observed in 5 and 10 min were similar to the folded protein adsorption in Fig. 5a, being treated as non-specific adsorption. For 20 and 30 min incubations, the increasing variations complied with the tendency observed for the heat-denatured protein in Fig. 5, with a stronger signal for greater exposure. This tendency, however, returned after $40 \mathrm{~min}$ of incubation. The protein was denatured by heat before the incubation, being able to refold after cooling. For the extensive incubations of 40 and $50 \mathrm{~min}$, the previously exposed residues were internalized due to protein refolding. Again, the folded conformation of the protein appeared to contribute to slow electron transfer between the interface of the electrolyte and the electrode. Furthermore, incubation for $30 \mathrm{~min}$ provided the most precise result $(\mathrm{RSD}=1.79 \%, \mathrm{n}=3$ ).

After each analysis, the sensor was washed with plenty of water, and immediately reused for sequential analysis with a higher concentration. The same sensor was used during six sequential analyses of increasing concentrations of protein, with good performance. Although the enzymatic removal step may damage the imprinted cavities, the analytical performance of the sensor along with the characterization data provides proof that the imprinted cavities are capable of successfully binding the protein. It seems that we initially employed incubation conditions that favor the formation of the cavities without much damage to the film.

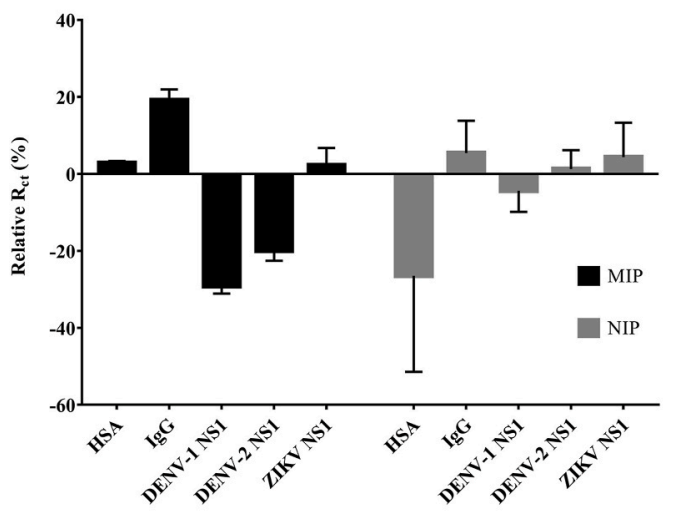

Nonetheless, we did not optimize this parameter.

\subsection{Selectivity and specificity of the sensor}

The influence of components from a real serum sample matrix was evaluated by incubation of MIP and NIP with HSA and IgG. Specificity was appraised by the response of the sensor to the NS1 proteins of DENV-2 and ZIKV. Spiked samples of diluted human serum (1:1000) were also tested by the sensor and the results are displayed in Fig. 6 . The responses observed for the NIP were attributed to non-specific adsorption, related to the affinity of the analytes tested to the chemical composition of the network. The small cavities of MIP appear to make the non-specific adsorption of HSA difficult, while the adsorption of IgG showed no statistical significance between MIP and NIP. DENV-2 NS1 displayed a signal similar to the response to DENV-1 NS1. Eight of the nine residues of the imprinted sequence are conserved among the four serotypes of DENV. This high degree of identity makes it difficult to distinguish the protein between serotypes. For ZIKV, however, no more than three residues of the sequence are conserved. This difference allowed the imprinted sensor to distinguish the ZIKV protein from DENV serotypes. As depicted in Fig. 6, the response was the same for NIP and MIP, with the variation observed being attributed to non-specific interaction. Overall, the sensor was able to provide a good analytical performance, showing good linearity $(\mathrm{r}=0.9678)$ and precision at the lowest concentration $\left(25 \mu \mathrm{g} \mathrm{L}{ }^{-1}, \mathrm{RSD}=15.96 \%\right)$. Besides that, the sensor presented almost the same sensitivity observed in PBS medium ( 0.1910 and 0.1915 , respectively), with RSD below $5.04 \%$ from 50 to

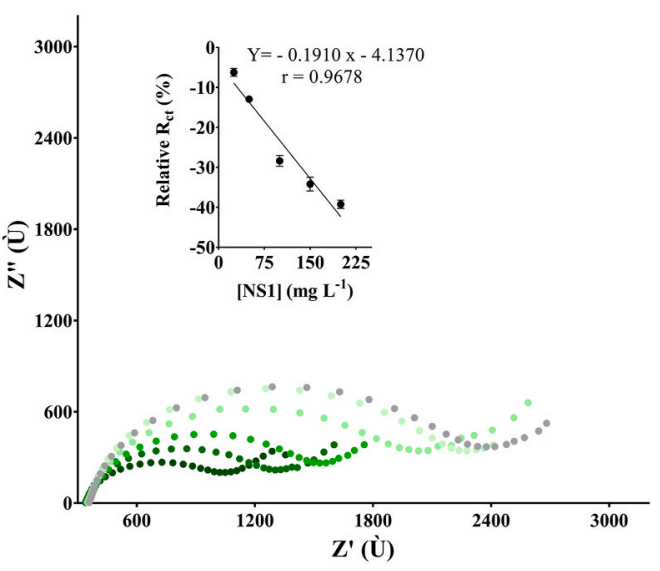

Fig. 6. Left: response obtained for peptide template against heat-denatured interferents (HSA and IgG) and heat-denatured NS1 proteins of DENV-1 and 2 and ZIKV. All analytes were incubated in $10 \times 10^{-3} \mathrm{~mol} \mathrm{~L}^{-1} \mathrm{PBS}$, pH $7.4\left(100 \mu \mathrm{g} \mathrm{L}^{-1}\right)$. Right: EIS calibration curve for DENV-1 NS1 protein rebinding in spiked samples of diluted human serum (1:1000). The reading for stabilized sensor (gray) is followed by that for rising concentrations of the protein, from 25 to $200 \mu \mathrm{g} \mathrm{L}^{-1}$. 
$200 \mu \mathrm{g} \mathrm{L}^{-1}$, thereby confirming the negligible effect of serum. The calculated LOD and LOQ for the diluted serum rebinding experiment were 29.3 and $88.7 \mu \mathrm{g} \mathrm{L}^{-1}$.

\section{Conclusions}

This work introduces a multidisciplinary approach for rational epitope design, considering conservational and structural properties and the immunogenicity of the sequence. Nonetheless, we provide a proof of concept for in situ exposure of the hidden sites of proteins without chemical modifications. The combination of these techniques provides a versatile protocol which can be applied for epitope imprinting for various MIP applications. These strategies were associated with direct electrochemical transduction, leading to a new state of art for sensors based on plastic antibodies and specifically epitope imprinting. The heat-denaturation step also dissolves previously formed antigen-antibody complexes. This can aid the avoidance of false negative results due to antibody cross-reactivity. Despite the analytical performance obtained in this work being inferior to that in the previous work of Arshad et al. (2020) ( $\left.\mathrm{LOD}=0.3 \mu \mathrm{g} \mathrm{L}^{-1}\right)$ and Tai (2006) ( $\left.\mathrm{LOD} \sim 1-10 \mu \mathrm{g} \mathrm{L}^{-1}\right)$, it attested the suitability of the sensor to perform a label-free differential diagnosis between DENV and ZIKV. This is a huge achievement, since the closely related symptoms of zika and dengue and the high degree of sequence homology exhibited among flaviviruses make differential diagnosis a difficult task even nowadays.

To overcome the difficulties found in this experimental model, some strategies can be suggested as future trends. Since non-specific adsorption can hardly be distinguished from specific adsorption in electrochemical sensing of unlabeled antigens (Ramanaviciene and Ramanavicius, 2004), the use of strong interactions between the polymer and analyte can boost the selectivity and specificity of the sensor. As an example, reversible covalent bonds (e.g. boronate-based systems) (Silva et al., 2020) aimed at glycosylation sites could be strong enough to hold the analyte during intense washing after incubation, enhancing the removal of interferents before the analysis. At the same time, the $\mathrm{pH}$-reversible character of this bond could also allow a mild regeneration process and several reuses.

Overall, this approach may open new horizons onto a novel imprinting approach for complex proteins, by using internal epitopes as templates. This will contribute to provide responses that are more specific and thereby reduce false negative/positive data.

\section{CRediT authorship contribution statement}

Matheus Siqueira Silva: Conceptualization, Methodology, Validation, Writing - review \& editing, Investigation. Ana Patricia Moreira Tavares: Methodology, Validation. Luiz Felipe Leomil Coelho: Conceptualization, Supervision, Writing - review \& editing, Investigation. Lígia Ely Morganti Ferreira Dias: Conceptualization, Methodology. Rosa Maria Chura-Chambi: Methodology, Conceptualization. Flávio Guimarães da Fonseca: Methodology, Conceptualization. Maria Goreti Ferreira Sales: Conceptualization, Supervision, Writing review \& editing, Investigation. Eduardo Costa Figueiredo: Conceptualization, Supervision, Writing - review \& editing, Investigation.

\section{Declaration of competing interest}

The authors declare that they have no known competing financial interests or personal relationships that could have appeared to influence the work reported in this paper.

\section{Acknowledgments}

This work was supported by the Fundação de Amparo à Pesquisa do Estado de Minas Gerais (FAPEMIG, Belo Horizonte, Brazil) [project CDSAPQ-00638-17]; the Conselho Nacional de Desenvolvimento Científico e
Tecnológico (CNPq, Brasília, Brazil) [project 301564/2019-1, 427365/ 2018-0]; and the Coordenação de Aperfeiçoamento de Pessoal de Nível Superior (CAPES) for financial support ("Matheus Siqueira Silva/ PPGCF/Processo no. 88881.133746/2016-01”).

\section{Appendix A. Supplementary data}

Supplementary data to this article can be found online at https://doi. org/10.1016/j.bios.2021.113419.

\section{References}

Akey, D.L., Brown, W.C., Dutta, S., Konwerski, J., Jose, J., Jurkiw, T.J., DelProposto, J., Ogata, C.M., Skiniotis, G., Kuhn, R.J., Smith, J.L., 2014. Science 343, 881-885. https://doi.org/10.1126/science.1247749.

Anusha, J.R., Kim, B.C., Yu, K.H., Raj, C.J., 2019. Biosens. Bioelectron. 142, 111511. https://doi.org/10.1016/j.bios.2019.111511.

Arshad, R., Rhouati, A., Hayat, A., Nawaz, M.H., Yameen, M.A., Mujahid, A., Latif, U., 2020. Appl. Biochem. Biotechnol. 191, 1384-1394. https://doi.org/10.1007/ s12010-020-03285-y.

Blacksell, S.D., 2012. J. Biomed. Biotechnol. 2012 https://doi.org/10.1155/2012 151967.

Bossi, A.M., Sharma, P.S., Montana, L., Zoccatelli, G., Laub, O., Levi, R., 2012. F Anal. Chem. 84, 4036-4041. https://doi.org/10.1021/ac203422r.

Braga, J.U., Bressan, C., Dalvi, P.R., Calvet, G.A., Daumas, R.P., Rodrigues, N., Wakimoto, M., Nogueira, R.M.R., Nielsen-Saines, K., Brito, C., Filippis, M.B. de, Brasil, P., 2017. PloS One 12, 1-14. https://doi.org/10.1371/journal.pone.0179725.

Bulusheva, L.G., Okotrub, A.V., Kinloch, I.A., Asanov, I.P., Kurenya, A.G., Kudashov, A. G., Chen, X., Song, H., 2008. Phys. Status Solidi Basic Res. 245, 1971-1974. https:// doi.org/10.1002/pssb.200879592.

Darwish, N.T., Alias, Y.B., Khor, S.M., 2015. Trends Anal. Chem. 67, 45-55. https://doi, org/10.1016/j.trac.2015.01.005.

Emini, E.A., Hughes, J.V., Perlow, D.S., Boger, J., 1985. Am. Soc. Microbiol. 55, 836-839.

Erdőssy, J., Horváth, V., Yarman, A., Scheller, F.W., Gyurcsányi, R.E., 2016. Trends Anal. Chem. 79, 179-190. https://doi.org/10.1016/j.trac.2015.12.018.

Ertürk, G., Mattiasson, B., 2017. Sensors 17, 1-17. https://doi.org/10.3390/s17020288.

Falconar, A.K.I., Young, P.R., Miles, M.A., 1994. Arch. Virol. 137, 315-326. https://doi. org/10.1007/BF01309478.

Ferrari, A.C., Meyer, J.C., Scardaci, V., Casiraghi, C., Lazzeri, M., Mauri, F., Piscanec, S., Jiang, D., Novoselov, K.S., Roth, S., Geim, A.K., 2006. Phys. Rev. Lett. 97, 1-4. https://doi.org/10.1103/PhysRevLett.97.187401.

Frasco, M., Truta, L., Sales, M., Moreira, F., 2017. Sensors 17, 523. https://doi.org/ $10.3390 /$ s17030523.

Gasco, S., Muñoz-Fernández, M.Á., 2020. Int. J. Mol. Sci. 22, 35. https://doi.org/ 10.3390/ijms22010035.

Gelanew, T., Poole-Smith, B.K., Hunsperger, E., 2015. J. Virol. Methods 1, 1-10. https:// doi.org/10.1016/j.jviromet.2015.06.003.

Karplus, P.A., Schulz, G.E., 1985. Naturwissenschaften 72, 212-213. https://doi.org/ 10.1007/BF01195768.

Kawashima, Y., Katagiri, G., 1995. Phys. Rev. B 52, 10053-10059. https://doi.org/ 10.1103/PhysRevB.52.10053.

Khan, M.A.R., Moreira, F.T.C., Riu, J., Sales, M.G.F., 2016. Sensor. Actuator. B Chem. 233, 697-704. https://doi.org/10.1016/j.snb.2016.04.075.

Khristunova, E., Dorozhko, E., Korotkova, E., Kratochvil, B., Vyskocil, Vlastimil, Barek, J., 2020. Sensors 20, 4600. https://doi.org/10.3390/s20164600.

Langerak, T., Mumtaz, N., Tolk, V.I., van Gorp, E.C.M., Martina, B.E., Rockx, B., Koopmans, M.P.G., 2019. PLoS Pathog. 15, e1007640 https://doi.org/10.1371/ journal.ppat.1007640.

Larsen, J.E.P., Lund, O., Nielsen, M., 2006. Immunome Res. 7, 1-7. https://doi.org/ 10.1186/1745-7580-2-2.

Luo, X., Davis, J.J., 2013. Chem. Soc. Rev. 42, 5944-5962. https://doi.org/10.1039/ c3cs60077g.

Mujahid, A., Mustafa, G., Dickert, F., 2018. Biosensors 8, 52. https://doi.org/10.3390/ bios8020052.

Pierson, T.C., Diamond, M.S., 2013. Flaviviruses. In: Cohen, J.I., Griffin, D.E., Lamb, R. A., Martin, M.A., Racaniello, V.R., Roizman, B. (Eds.), Fields Virology V. I. Lippincott Williams \& Wilkins, Philadelphia, pp. 747-794.

Ramanaviciene, A., Ramanavicius, A., 2004. Biosens. Bioelectron. 20, 1076-1082. https://doi.org/10.1016/j.bios.2004.05.014.

Ramanavicius, S., Ramanavicius, A., 2021. Polymers 13, 1-19. https://doi.org/10.3390/ polym13010049.

Ribeiro, J.A., Pereira, C.M., Silva, A.F., Sales, M.G.F., 2017. Anal. Chim. Acta 981, 41-52. https://doi.org/10.1016/j.aca.2017.05.017.

Rosa Da Silva, C.M., Chura-Chambi, R.M., Ramos Pereira, L., Cordeiro, Y., De Souza Ferreira, L.C., Morganti, L., 2018. BMC Biotechnol. 18, 1-10. https://doi.org/ 10.1186/s12896-018-0486-2.

Roscher, S., Hoffmann, R., Ambacher, O., 2019. Anal. Methods 11, 1180-1191. https:// doi.org/10.1039/c8ay02619j.

Saylan, Y., Yilmaz, F., Özgür, E., Derazshamshir, A., Yavuz, H., Denizli, A., 2017. Sensors 17. https://doi.org/10.3390/s17040898. 
Silva, M.S., Tavares, A.P.M., de Faria, H.D., Sales, M.G.F., Figueiredo, E.C., 2020. Crit. Rev. Anal. Chem. 1-16. https://doi.org/10.1080/10408347.2020.1843131.

Tai, D.-F., 2006. Clin. Chem. 52, 1486-1491. https://doi.org/10.1373/ clinchem.2005.064501.

Tai, D.F., Lin, C.Y., Wu, T.Z., Chen, L.K., 2005. Anal. Chem. 77, 5140-5143. https://doi. org/10.1021/ac0504060.

Wilder-Smith, A., 2018. Curr. Infect. Dis. Rep. 20, 50. https://doi.org/10.1007/s11908 018-0656-3.
Winkler, G., Maxwell, S.E., Ruemmler, C., Stollar, V., 1989. Virology 171, 302-305. https://doi.org/10.1016/0042-6822(89)90544-8.

Winkler, G., Randolph, V.B., Cleaves, G.R., Ryan, T.E., Stollar, V., 1988. Virology 162, 187-196. https://doi.org/10.1016/0042-6822(88)90408-4.

Zahedi, P., Ziaee, M., Abdouss, M., Farazin, A., Mizaikoff, B., 2016. B polym. Adv. Met. Technol. 2016 https://doi.org/10.1002/pat.3754 n/a-n/a. 